\begin{tabular}{|c|l|}
\hline Title & Potential A pplication of Poly(N-isopropylacrylamide) Gel Containing Polymeric Micelles to Drug Delivery Systems \\
\hline Author(s) & Y an, Hu; Tsujii, Kaoru \\
\hline Citation & $\begin{array}{l}\text { Colloids and Surfaces B: Biointerfaces, 46(3), 142-146 } \\
\text { https://doi.org/10.1016/.colsurfb.2005.10.007 }\end{array}$ \\
\hline Issue Date & 2005 \\
\hline Doc URL & http://hdl.handle.net/2115/1381 \\
\hline Type & article(author version) \\
\hline File Information & CSB-B46(3).pdf \\
\hline
\end{tabular}

Instructions for use 


\title{
Potential Application of Poly( $N$-isopropylacrylamide) Gel Containing Polymeric Micelles to Drug Delivery Systems
}

\author{
Hu Yan* and Kaoru Tsujii* \\ Nanotechnology Research Center, Research Institute for Electronic Science, Hokkaido University, \\ Sapporo 001-0021, Japan
}

\begin{abstract}
We have investigated rapidly thermo-responsive NIPA gel containing polymer surfactant PMDP (NIPA-PMDP gel) as a potential drug carrier using (+)-L-ascorbic acid as a model drug. In the NIPA-PMDP gel system micelles of polymer surfactant PMDP are trapped by the entanglement of polymer chains inside the gel networks. Therefore, in principle the gel system tightly stores targeted drug in the micelles and rapidly releases controlled amount of the drug by switching on-off of external stimuli such as temperature or infrared laser beam. In our investigation on release profile, the NIPA-PMDP gel system showed completely different releasing behavior from that of the conventional NIPA gel. The NIPA-PMDP gel released rapidly all loaded (+)-L-ascorbic acid above the phase transition temperature (ca. $34{ }^{\circ} \mathrm{C}$ ), while slowly released the corresponding amount of the drug below the temperature. In contrast, the conventional NIPA gel released more slowly limited amount of the drug above the phase transition temperature while similarly did to the NIPA-PMDP gel below the temperature. The release profile of the NIPA-PMDP gel seems to be governed by only kinetics of volume phase transition of the gel network but not by the hydrophobic domains of the micelles probably because of too hydrophilic nature of (+)-L-ascorbic acid.
\end{abstract}

Keywords: Thermoresponsive gel; Poly( $N$-isopropylacrylamide) (NIPA) gel; Polymer surfactant; Volume phase transition; Drug delivery system

\section{Introduction}

Poly( $N$-isopropylacrylamide) (NIPA) gel has attracted considerable attention from both academic and technological aspects [1-15]. NIPA

* Corresponding author. Tel.: +81 117069348 (H. Yan) or 9356 (K. Tsujii); fax: +81 11706 9357.

E-mail address: yanhu@es.hokudai.ac.jp (H. Yan) or tsujik@es.hokudai.ac.jp (K. Tsujii) gel undergoes an abrupt volume change at the phase transition temperature (ca. $\left.34{ }^{\circ} \mathrm{C}\right)$ [1]. The abrupt volume change can be utilized in promising application of drug delivery systems [9-15]. Several strategies have been reported to realize much more rapid volume change of NIPA gels for better application of them [5-8]. Recently we have created NIPA gel system containing 
polymer surfactant poly(2-(methacryloyloxyl) decylphosphate) (PMDP) (cf. Scheme 1) [16, 19] and have studied on bimorph-structured gel actuators [17]. We have also succeeded in synthesis of cylindrical microgels of the system [18]. The gel system shows rapid volume change by stimulus of temperature as well as laser beam irradiation [16]. Structurally the gel system contains the trapped micelles of PMDP inside the NIPA networks. Therefore, at least in principle the gel system tightly stores targeted drug in the micelles and rapidly releases controlled amount of the drug by switching on-off of external stimuli such as temperature or infrared laser beam. Herein we report on controlled-releasing profile of the NIPA-PMDP gel system using (+)-L-ascorbic acid and temperature as a targeted drug and a stimulus, respectively.

\section{Materials and methods}

Cylindrical NIPA-PMDP and NIPA gels were synthesized according to the procedure previously reported elsewhere [16]. The NIPA-based gels were synthesized by a free-radical polymerization as follows: $0.044 \mathrm{~g}$ of PMDP, $0.87 \mathrm{~g}$ of NIPA (cf. Scheme 1), $0.014 \mathrm{~g}$ of $N, N^{\prime}$-methylene bisacrylamide (MBA), and $0.013 \mathrm{~g}$ of ammonium persulfate (APS) were dissolved in $8.5 \mathrm{~g}$ of deionized water. The solution was adjusted to $\mathrm{pH}$ 6 using $0.93 \mathrm{~g}$ of $0.1 \mathrm{M} \mathrm{NaOH}$ solution, then

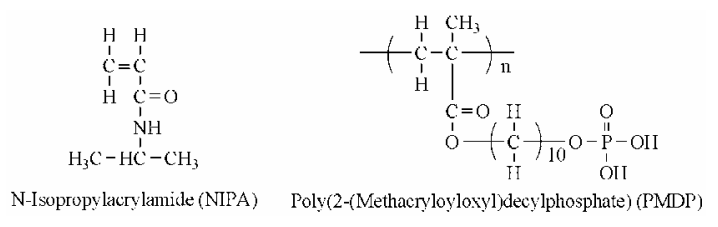

Scheme 1. Chemical structures of NIPA monomer and polymer surfactant PMDP. degassed and polymerized at $4{ }^{\circ} \mathrm{C}$ by the addition of $24 \mu \mathrm{l}$ of tetramethylenediamine (TEMED). Polymerizations were carried out in 1.3 mm-capillaries. The resulting NIPA-PMDP gels were repeatedly washed by immersion in pure water for several days. The NIPA gels were similarly synthesized but without PMDP.

Dye-solubilization experiment was carried out to confirm the presence of PMDP micelles in the gel. Yellow AB was chosen as an oil-soluble dye which is insoluble in water. Colors of the gels with and without addition of the Yellow $\mathrm{AB}$ were observed and recorded by an optical microscope (Olympus SZX7) with a digital camera.

Release profiles of the model drug from the gels were preliminarily measured as follows: Cylindrical NIPA-PMDP or NIPA gels with initial size of $1.7 \mathrm{~mm}$ diameter x $9.2 \mathrm{~mm}$ length were used for measurements of the release profiles. The cylindrical gels were immersed in the solution of (+)-L-ascorbic acid (0.28 wt\%) for $24 \mathrm{hr}$ at $9{ }^{\circ} \mathrm{C}$ to load the model drug. Then the gels were washed by rinsing to remove the model drug adhering on surface of the gels. The experiments of release profile were performed in a quartz cell $(1 \mathrm{~cm} \times 1 \mathrm{~cm})$ filled with pure water at room temperature $\left(24{ }^{\circ} \mathrm{C}\right)$ and above the phase transition temperature $\left(39 \sim 46{ }^{\circ} \mathrm{C}\right)$. The quartz cell in which the gels immersed was heated by thermoelectric heating/cooling unit (Netsu Denshi Thermomodule controller, MT862-04C12). The solution in the quartz cell was employed at a time interval for measurement of ultraviolet spectra by a spectrophotometer (Hitachi U-3010). The quartz cell was returned to the temperature-controlled water bath to keep it at the releasing temperature. 
The method should be improved further to keep the same temperature on UV measurement as that in water bath. The (+)-L-ascorbic acid has strong absorption at $205 \mathrm{~nm}$ of wavelength. Therefore, intensity of the absorption at $205 \mathrm{~nm}$ of wavelength has been measured for the solution in which the gel immersed. Finally cumulative amount of the (+)-L-ascorbic acid that the gel released was calculated by calibration with a standard concentration curve. The time-dependant cumulative amounts were normalized by the final cumulative one in the UV measurement.

After the preliminary investigation the release profiles were measured by HPLC technique under relatively well-controlled condition. The typical procedure was as follows: Cylindrical NIPA-PMDP gels with initial size of $1.7 \mathrm{~mm}$ diameter x $9.2 \mathrm{~mm}$ length were immersed in the solution of (+)-L-ascorbic acid (0.28 wt\%) for 5 days at $9{ }^{\circ} \mathrm{C}$ to load the model drug. Then the gels were washed by rinsing to remove the model drug adhering on the surface of the gels. The experiments of release profile were performed in a bottle filled with $10 \mathrm{~g}$ of pure water. The bottle in which the gels immersed was set in temperature-controllable $\left( \pm 0.1{ }^{\circ} \mathrm{C}\right)$ water-bath (EYELA NTT-2000). The solution in the bottle was sampled at a certain time interval at $27{ }^{\circ} \mathrm{C}$ and $40{ }^{\circ} \mathrm{C}$, respectively. The sampled solution was measured by a HPLC analytic system. The HPLC chromatographic conditions were as follows: HPLC analytic system, Hitachi ELITE LaChrom; ODS column at $40{ }^{\circ} \mathrm{C}$; flow rate, $1 \mathrm{ml} / \mathrm{min}$; eluent, distilled water (HPLC grade); wavelength of detection, 205 nm. Cumulative amount (mg) of the (+)-L-ascorbic acid that the gel has released was calculated by calibration with a standard concentration curve which was prepared using chromatographic peak at retention time of 1.28. In the HPLC method the time-dependant cumulative amount was not normalized.

\section{Results and discussion}

As previously reported [16], the NIPA-PMDP gel shows five-fold and four-fold greater water-absorbencies than simple NIPA gel in pure water and in $0.15 \mathrm{M} \mathrm{NaCl}$ solution, respectively. And the phase transition temperature of the NIPA-PMDP gel was interestingly comparable to that of the conventional NIPA gel, both in pure water and in $0.15 \mathrm{M} \mathrm{NaCl}$ solution. It is important to note that the NIPA gel has been extensively studied as a candidate of DDS mainly because its transition temperature is ca. $34{ }^{\circ} \mathrm{C}$, which is comparable to body temperatures [12].

The NIPA-PMDP gel also shows significantly rapid volume change $(\Delta \mathrm{V} \%)$ than that of NIPA gel at $43{ }^{\circ} \mathrm{C}[16]$. The NIPA-PMDP gel shows $\Delta \mathrm{V} \%$ of $88 \%$ within $30 \mathrm{~min}$, and subsequently collapsed within 120 min; in contrast, the NIPA

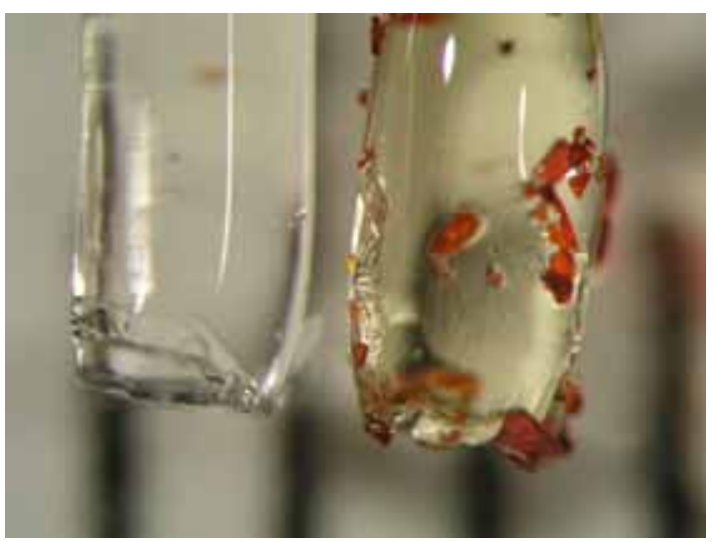

Figure 1. Cylindrical NIPA-PMDP gels with (right) and without (left) oil-soluble dye Yellow AB. Fine crystals of Yellow AB are attached to the gel in the right side figure. 
gel showed only $24 \%$ within $30 \mathrm{~min}$, and did not attain the completely collapsed state within the experimental time ( $2 \mathrm{hr})$. The rapid volume phase transition of the NIPA-PMDP gel is reproducible, reversible and repeatable. No release of PMDP molecule from the NIPA-PMDP gel system is observed during the reversible volume change, which is confirmed by ultraviolet absorption spectra [16]. The colorless NIPA-PMDP gel (left side of Figure 1) solubilizes an oil-soluble dye, Yellow AB, which is hydrophobic crystal and insoluble in water. The Yellow AB-solubilized NIPA-PMDP gel clearly showed yellowish color, as shown in right side of Figure 1. Furthermore

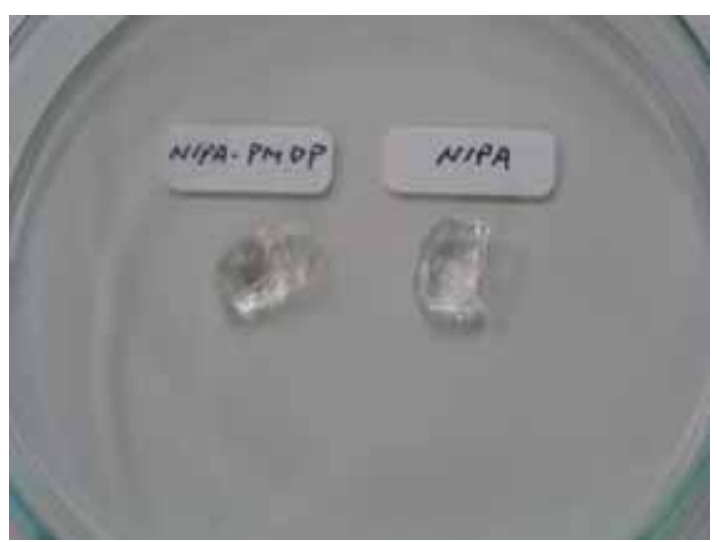

Figure 2. NIPA-PMDP (left side) and NIPA (right side) gels after immersing in water containing Yellnw AR nowder

the NIPA gel did not show the yellowish color, as shown in Figure 2, even when the gel was immersed in the water containing the Yellow AB powder. These results indicate that PMDP forms micelles within the NIPA gel network, and the micelles of polymer surfactant are trapped inside the gel networks, as schematically illustrated in Figure 3.

Accordingly we can suggest novel drug delivery system by using the NIPA-PMDP gel. Theoretically the NIPA-PMDP gel system tightly stores targeted drug in the micelles and rapidly

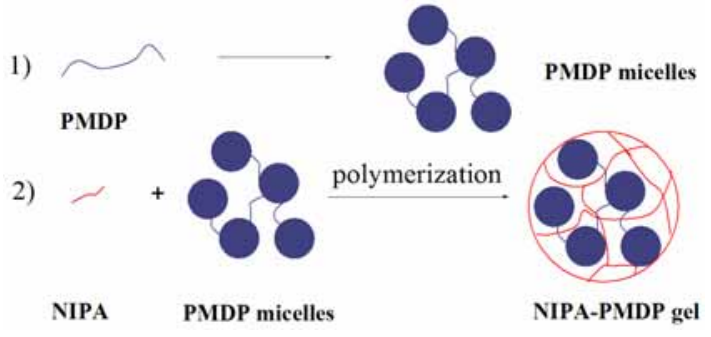

Figure 3. Schematic illustration of trapped micelles within the NIPA-PMDP gel system.

releases controlled amount of the drug by switching on-off of external stimuli such as temperature or infrared laser beam. The release profile may be completely different from the conventional NIPA gel.

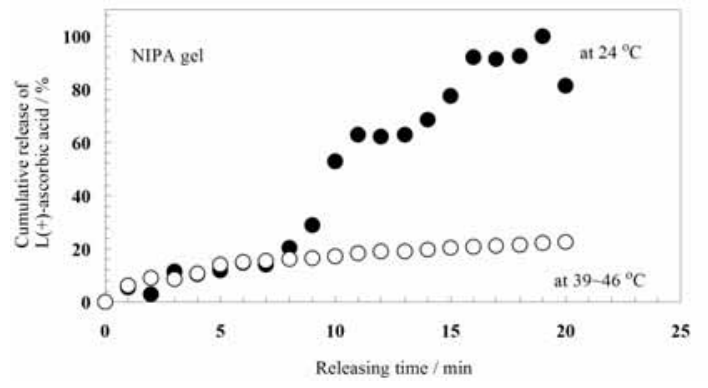

Figure 4. Release profiles (UV) of the NIPA gels above and below the phase transition temperature.

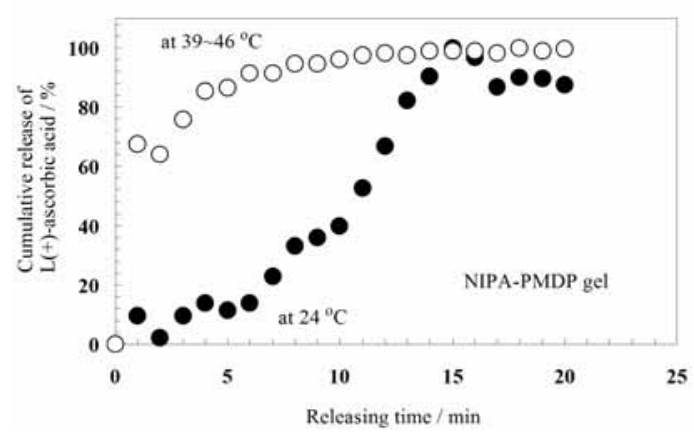

Figure 5. Release profiles (UV) of the NIPA-PMDP gels above and below the phase transition temperature.

The NIPA and NIPA-PMDP gels were used to obtain an on-off release profile of (+)-L-ascorbic acid in response to a temperature change as shown in Figures 4 and 5. The NIPA gel slowly released limited amount of the drug above the 
phase transition temperature while gradually released all loaded drug below the temperature (Figure 4). The slow release above the transition temperature is well interpreted by the formation of a skin-type barrier which is less permeable to

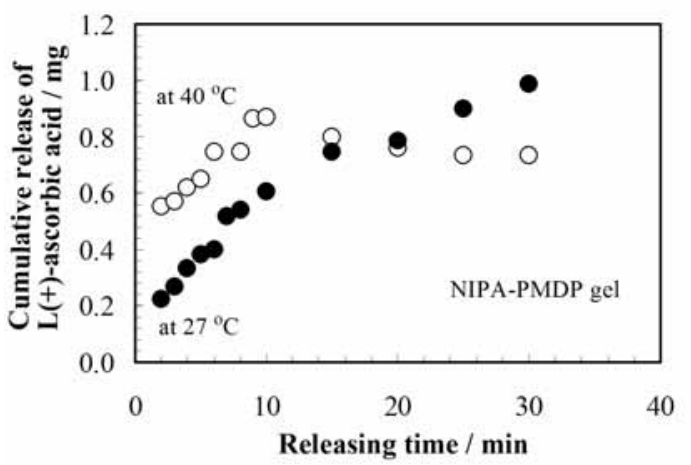

Figure 6. Release profiles (HPLC) of the NIPA-PMDP gels above and below the phase transition temperature.

the drug [12]. On the other hand, the NIPA-PMDP gel rapidly released all loaded (+)-L-ascorbic acid above the phase transition temperature (ca. $34{ }^{\circ} \mathrm{C}$ ), while showed slower releasing profile which is similar to that of the NIPA gel below the phase

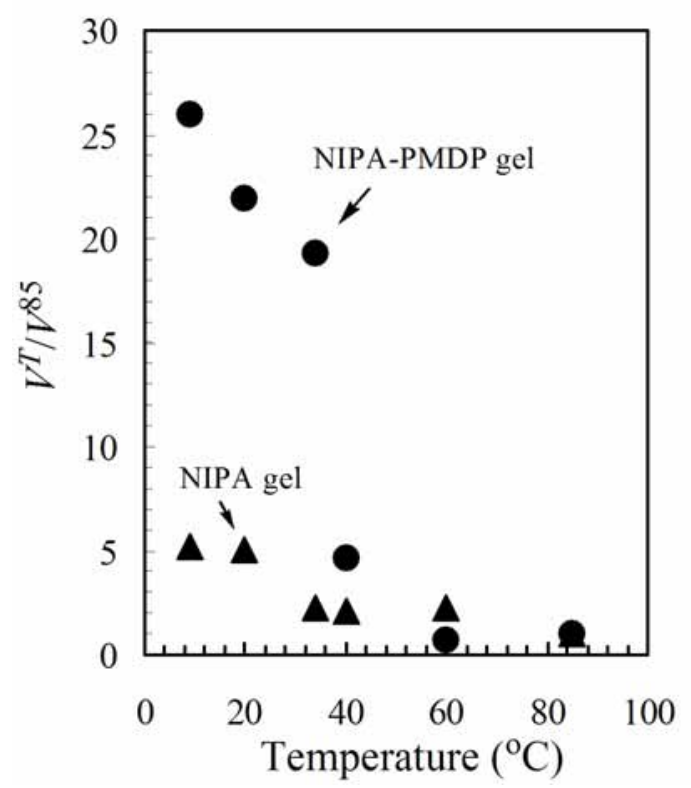

Figure 7. Volume phase transitions of the NIPA-PMDP and NIPA gels in water. $V^{T}$ and $V^{85}$ are volumes of the gels at target temperature and $85^{\circ} \mathrm{C}$, respectively (cf. Ref. 16). transition temperature (Figure 5). The release profile of the NIPA-PMDP measured by the HPLC analytic technique was shown in Figure 6. The NIPA-PMDP gel released the $\mathrm{L}(+)$-ascorbic acid slowly and gradually with time at $27{ }^{\circ} \mathrm{C}$ which is lower than the phase transition temperature, $34{ }^{\circ} \mathrm{C}$ (Figure 6). The gel did not stop the release within the investigated time scale. On the other hand, the gel rapidly release the drug and finished the release within $10 \mathrm{~min}$ at $40{ }^{\circ} \mathrm{C}$ which is higher than the phase transition temperature. It is noted that, first the loading amount of the drug in the single gel was not exactly the same, and secondly the concentration of the sampled solution was somehow different from that of the completely mixed solution of the released drug. Therefore the experimental method needs further improvement to probe precisely and quantitatively the release profile of the NIPA-PMDP gels. Although some differences induced methodologically were observed in detail, the releasing trend was quite similar each other in Figures 5 and 6.

The rapid release above the transition temperature was explained by the rapid volume phase transition of the gel system [16], as shown in Figure 7. The NIPA-PMDP gel showed drastic volume change during the phase transition while the NIPA gel showed much less volume change at the same temperature range since well-known skin formation. The difference of release profiles between the NIPA and the NIPA-PMDP gels above the temperature exactly reflected the difference of the volume change during the phase transition [16]. The similar release profile below the transition temperature was suggested that the 
(+)-L-ascorbic acid (cf. Scheme 2) is loaded inside the NIPA-PMDP gel network but outside the micelles of the polymer surfactant PMDP since the drug is highly hydrophilic, and the inner

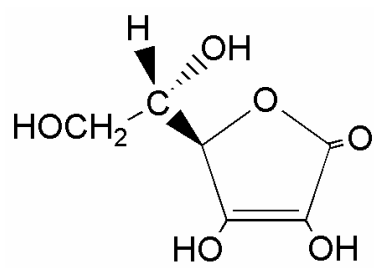

Scheme 2. Chemical structure of the $(+)$-L-ascorbic acid.

domain of the micelles is highly hydrophobic.

It is noted that the release profile should be investigated in physiologic media, and the phase transition temperature of the NIPA-PMDP gel should be lifted above body temperature in the case of practical applications.

In conclusion, the rapidly thermo-responsive NIPA-PMDP gel system, in which micelles of polymer surfactant PMDP are trapped within the gel network, showed completely different releasing behavior from the conventional NIPA gel. The NIPA-PMDP gel rapidly released all loaded (+)-L-ascorbic acid above the phase transition temperature (ca. $34{ }^{\circ} \mathrm{C}$ ), while slowly released the corresponding amount of the drug below the temperature. In contrast, the conventional NIPA gel more slowly released limited amount of the drug above the temperature while similarly released with the NIPA-PMDP gel below the temperature. The release profile of the NIPA-PMDP gel may be governed by only the kinetics of volume phase transition of the gel network but not by the hydrophobic domains of the micelles probably because of too hydrophilic nature of the (+)-L-ascorbic acid. The
NIPA-PMDP gel system may be suitable for a drug delivery system which rapidly release controlled amount of some hydrophobic drug by on-off of external stimuli such as infrared laser irradiation.

\section{Acknowledgement}

We acknowledge financial support from $21^{\text {st }}$ century COE program "Center of Excellence for Advanced Life Science on the Base of Bioscience and Nanotechnology” of Hokkaido University, and the Grant-in-Aid for Scientific Research from Ministry of Education, Culture, Sports, Science, and Technology, Japan.

\section{References}

[1] Y. Hirokawa, T. Tanaka, Volume phase transition in a nonionic gel, J. Chem. Phys. 81 (1984) 6379-6380.

[2] Y. Zhang, T. Tanaka, M. Shibayama, Super-absorbency and phase transition of gels in physiological salt solutions, Nature 360 (1992) 142-144.

[3] S. Miyagishi, M. Takagi, S. Kadono, A. Ohta, T. Asakawa, Effect of amino acid surfactants on phase transition of poly( $\mathrm{N}$-isopropylacrylamide) gel, J. Colloid Interface Sci. 261 (2003) 191-196. [4] T. Okajima, I. Harada, K. Nishio, S. Hirotsu, Kinetics of volume phase transition in poly(N-isopropylacrylamide) gels, J. Chem. Phys. 116 (2002) 9068-9077.

[5] X. Zhang, R. Zhuo, Y. Yang, Using mixed solvent to synthesized temperature sensitive poly(N-isopropylacrylamide) gel with rapid 
dynamics properties, Biomaterials 23 (2002) 1313-1318.

[6] W. Xue, I. W. Hamley, M. B. Huglin, Rapid swelling and deswelling of thermoreversible hydrophobically modified poly(N-isopropylacrylamide) hydrogels prepared by freezing polymerization, Polymer 43 (2002) 5181-5186.

[7] R. Yoshida, K. Uchida, Y. Kaneko, K. Sakai, A. Kikuchi, Y. Sakurai, T. Okano, Comb-type grafted hydrogels with rapid deswelling response to temperature changes, Nature 374 (1995) 240-242. [8] T. Matsuura, M. Sugiyama, M. Annaka, Y. Hara, T. Okano, Microscopic implication of rapid shrinking of comb-type grafted poly( $N$-isopropylacrylamide) hydrogels, Polymer 44 (2003) 4405-4409.

[9] I. Kwon, Y. Bae, S. Kim, Electrically credible polymer gel for controlled release of drugs, Nature 354 (1991) 291-293.

[10] R. Yoshida, K. Sakai, T. Okano, Y. Sakurai, Pulsatile drug delivery systems using hydrogels, Adv. Drug Delivery Rev. 11 (1993) 85-108.

[11] B. Jeong, Y. Bae, D. Lee, S. Kim, Biodegradable block copolymers as injectable drug-delivery systems, Nature 388 (1997) 860-862.

[12] L. E. Bromberg, E. S. Ron, Temperature-responsive gels and thermogelling polymer matrices for protein and peptide delivery, Adv. Drug Delivery Rev. 31 (1998) 197-221.

[13] Y. Qiu, K. Park, Environment-sensitive hydrogels for drug delivery, Adv. Drug Delivery Rev. 53 (2001) 321-339.

[14] M. Ishida, H. Sakai, S. Sugihara, S. Aoshima, S. Yokoyama, M. Abe, Controlled release of vitamin E from thermo-responsive polymeric physico-gel, Chem. Pharm. Bull. (Tokyo) 51 (2003) 1348-1349.

[15] X. Zhang, P. J. Lewis, C. Chu, Fabrication and characterization of a smart drug delivery system: microsphere in hydrogel, Biomaterials, 26 (2005) 3299-3309.

[16] H. Yan, H. Fujiwara, K. Sasaki, K. Tsujii, Rapid swelling/collapsing behaviors of thermo-responsive poly( $N$-isopropylacrylamide) gel containing poly(2-(methacryloyloxy)decylphosphate) surfactant, Angew. Chem. Int. Ed. 44 (2005) 1951-1954.

[17] H. Yan, K. Tsujii, Novel Bimorph-Structured Hydrogel Containing Segregated Polymer Surfactant, Polym. J. 37 (2005) 858-861.

[18] H. Yan, M. Nishino, Y. Tsuboi, N. Kitamura, K. Tsujii, Template-Guided Synthesis and Individual Characterization of Poly(N-isopropylacrylamide)-Based Microgels, Langmuir 21 (2005) 7076-7079.

[19] T. Koudate, Y. Nakano, JP patent P2000-26481A (2000). 